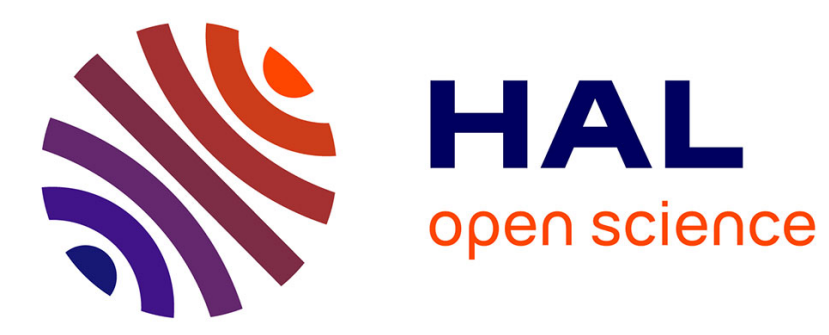

\title{
Modeling and stability of balloon-borne gondolas with coupled pendulum-torsion dynamics
}

Ervan Kassarian, Francesco Sanfedino, Daniel Alazard, Hélène Evain, Johan Montel

\section{- To cite this version:}

Ervan Kassarian, Francesco Sanfedino, Daniel Alazard, Hélène Evain, Johan Montel. Modeling and stability of balloon-borne gondolas with coupled pendulum-torsion dynamics. Aerospace Science and Technology, 2021, 112, pp.0. 10.1016/j.ast.2021.106607 . hal-03273935

\section{HAL Id: hal-03273935 \\ https://hal.science/hal-03273935}

Submitted on 29 Jun 2021

HAL is a multi-disciplinary open access archive for the deposit and dissemination of scientific research documents, whether they are published or not. The documents may come from teaching and research institutions in France or abroad, or from public or private research centers.
L'archive ouverte pluridisciplinaire HAL, est destinée au dépôt et à la diffusion de documents scientifiques de niveau recherche, publiés ou non, émanant des établissements d'enseignement et de recherche français ou étrangers, des laboratoires publics ou privés. 


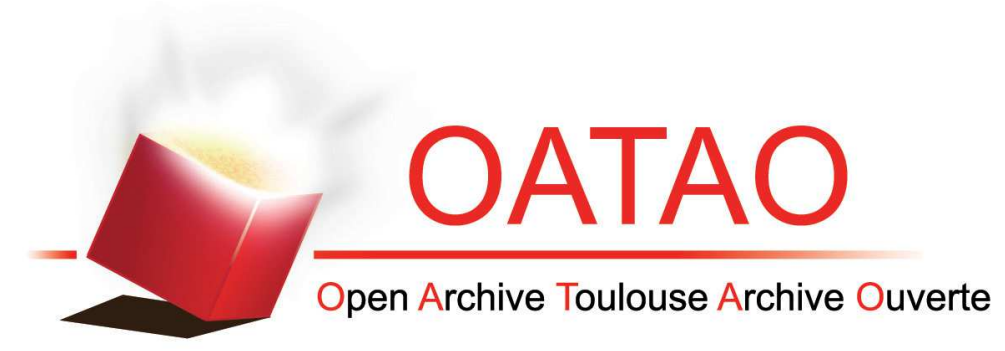

\section{Open Archive Toulouse Archive Ouverte (OATAO)}

OATAO is an open access repository that collects the work of some Toulouse researchers and makes it freely available over the web where possible.

This is an author's version published in: https://oatao.univ-toulouse.fr/28075

Official URL:https://doi.org/10.1016/j.ast.2021.106607

\section{To cite this version :}

Kassarian, Ervan and Sanfedino, Francesco and Alazard, Daniel and Evain, Hélène and Montel, Johan Modeling and stability of balloon-borne gondolas with coupled pendulum-torsion dynamics. (2021) Aerospace Science and Technology, 112. ISSN 1270-9638

Any correspondence concerning this service should be sent to the repository administrator: tech-oatao@listes-diff.inp-toulouse.fr 


\title{
Modeling and stability of balloon-borne gondolas with coupled pendulum-torsion dynamics
}

\author{
E. Kassarian ${ }^{\mathrm{a}, *}$, F. Sanfedino $^{\text {a }}$, D. Alazard ${ }^{\mathrm{a}}$, H. Evain ${ }^{\mathrm{b}}$, J. Montel $^{\mathrm{b}}$ \\ a ISAE-SUPAERO, 10 Avenue Edouard Belin, 31055 Toulouse, France \\ b CNES, 18 Avenue Edouard Belin, 31055 Toulouse, France
}

Keywords:

Stratospheric balloons

Pendulum-torsion coupling

Dynamic modeling

Stability

\begin{abstract}
A B S T R A C T
The objective of this paper is to fill the gap in literature on an exhaustive coupled pendulum-torsion model for balloon-borne systems. The development of such a model is required to explain the unexpected oscillatory behavior recorded on the flight data of scientific balloon-borne missions and more particularly the performance degradation due to the coupling of pendulum and azimuth dynamics through the azimuth control loop, which is classically designed using a decoupled torsion model. First, a complete dynamic model of balloon-borne systems is derived. The proposed model is applied to the Faint Intergalactic-medium Redshifted Emission Balloon (FIREBall) experiment and validated by flight data. Then, the stability issue raising from the commonly neglected coupling assumption is investigated. Sufficient stability conditions are presented by using positivity properties. Based on the FIREBall model, it is finally shown how the azimuth control can destabilize the pendulum dynamics, and how the proposed model can be used during preliminary design phases to size a flight chain and the associated control system to prevent this instability.
\end{abstract}

\section{Introduction}

Stratospheric balloons have been used for many years as platforms to carry scientific payloads into upper layers of the atmosphere. Compared to satellites and launchers, they are cost efficient, reusable, flexible in their deployment and operability, able to carry heavy payloads, and impose few mechanical constraints on their payload [1, chap. 1.3]. Allowing in-situ observations of the atmosphere as well as telescopic observations of the sky, they have enabled valuable science in various astronomy and Earth science disciplines [2]. For many applications, the pointing requirements become more and more challenging. Current architectures are able to provide telescope stability under the arc-second [3,4], and current developments aim at further improving line of sight precision to the milli-arcsecond by using fast-steering or deformable mirrors $[5,6]$. Thus, an accurate dynamic modeling of ballon-borne flight chains and the development of specific control algorithms are necessary.

Two types of dynamics are generally distinguished in the modeling of balloon systems. About the vertical axis, the torsion of

\footnotetext{
* Corresponding author.

E-mail addresses: ervan.kassarian@isae-supaero.fr (E. Kassarian), francesco.sanfedino@isae-supaero.fr (F. Sanfedino), daniel.alazard@isae-supaero.fr (D. Alazard), helene.evain@cnes.fr (H. Evain), johan.montel@cnes.fr (J. Montel).
}

the flight chain is traditionally modeled as a mass-spring system [7-15]. Although the stiffness is generally experimentally determined by system identification, which requires to deploy the whole system and process in-flight data, the bifilar pendulum model allows to analytically derive the stiffness of balloon flight chains $[16,17]$. About the two other axes, balloon flight trains experience a dynamic behavior typical of a floating multiple pendulum, which can be modeled with Lagrange's mechanics $[11,18]$ or multi-body approaches $[19,20]$. These dynamics have received less attention in the literature, and even when they are modeled, the azimuth control is most often designed by considering only a model of the torsion of the flight train around the vertical axis.

However, the loading of the gondola is rarely symmetrical, which leads to a coupling between the azimuth of the gondola and the pendulum dynamics. The presence of common resonance peaks in the spectral analysis of the gondola's attitude, reflecting their dynamical coupling, was observed in [21,3]. In the design of azimuth controllers, with no modeling of the pendulum oscillations, this coupling has sometimes been considered as neglected dynamics [7] or a parasite torque with unknown gain and frequency [10]. Indeed, the azimuth control of the gondola excites the pendulum modes, and reciprocally, the pendulum oscillations trigger the azimuth control. Thus, the pendulum modes can also be destabilized by the azimuth control. This phenomenon has been suspected during the Carmen flight in 2017 and during the Polar- 
ized Instrument for Long wavelength Observation of the Tenuous interstellar medium (PILOT) experiments in 2015 and 2017. Furthermore, the pendulum modes may be crucial in the pointing performance, as observed in the Faint Intergalactic-medium Redshifted Emission Balloon (FIREBall) experiment in 2018 [3], both for the coarse pointing (azimuth of the gondola) and fine pointing (on-board instrument). All these observations justify the need for a new modeling of the coupling between the gondola's azimuth and the pendulum oscillations, and an analysis of the consequences on the closed-loop system. It is also worth noticing that the experimental validation of balloon-borne flight chain models requires to deploy the whole system, which cannot be done in laboratory. As a consequence, the available flight data is often limited, since it is only acquired during the science missions. To the best of the authors' knowledge, the matching of the eigenmodes predicted by the model with the modes observed in flight has never been performed in the literature, although $[20,22]$ discuss the spectral analysis of flight data.

In this paper, we consider typical stratospheric balloon-borne missions. Once a steady altitude is reached $(20 \mathrm{~km}$ to $50 \mathrm{~km}$, depending on the mission), the attitude control system is turned on and the platform must provide pointing stability during a few hours. This work focuses on the dynamics of the balloon, flight train and gondola during this pointing phase. We propose to derive a new coupled pendulum-torsion model for balloon-borne flight trains, and to investigate the consequences of this coupling on the stability of the closed-loop system. The modeling, based on Lagrangian mechanics, is proposed in Section 2. The method is applied to the Faint Intergalactic-medium Redshifted Emission Balloon (FIREBall) experiment [3,23]. The predicted natural frequencies are compared to the spectral analysis of the flight data to validate the model. Then, the closed-loop stability is studied with the positivity framework $[24,25]$ in Section 3. A sufficient condition is derived to ensure the asymptotic stability of the torsionazimuth model, regardless of the parameters of the flight train. It is demonstrated how the stability of the torsion-azimuth system is no longer guaranteed when considering the coupling with the pendulum dynamics and how it can be recovered by damping the pendulum oscillations with a reaction wheel. A numerical application finally shows how the proposed model can detect and correct the instability of one of the pendulum modes of a system when the azimuth control is designed only on the torsion-azimuth dynamic model.

\section{Dynamical modeling}

\subsection{Pendulum dynamics modeling}

Let us consider the scheme of a typical balloon architecture in Fig. 1. The dynamics in the plane $(\mathbf{y}, \mathbf{z})$ are first considered, given a flight chain composed of $n+1$ bodies, where body 1 is the balloon and body $n+1$ is the gondola. For each body $k$, let $L_{k}$ be its length, $\rho_{k}(\in[0,1])$ the normalized position of its center of gravity from the top end of the body, $m_{k}$ its mass, $I_{k}^{X}$ its moment of inertia around $\mathbf{x}$ at the center of gravity. The distance between the center of gravity and the center of buoyancy of the balloon is $\lambda L_{1}$. The center of gravity is typically higher than the center of buoyancy, since the envelop is reinforced at its top end to resist the buoyant force. The angle of each body with respect to an inertial reference frame is noted $\theta_{k}^{x} \cdot y_{k}$ and $z_{k}$ are the variations of position of the center of gravity of the body $k$. Each body is subject to its weight $m_{k} \mathbf{g}$. The system is assumed at an equilibrium altitude, with the buoyant force being equal in norm to the total weight of the system $\sum_{i}^{n+1} m_{i}$ g. This assumption is legitimate because, during the pointing phase of the mission, (i) the altitude variations have low amplitude and are much slower than the dynamics

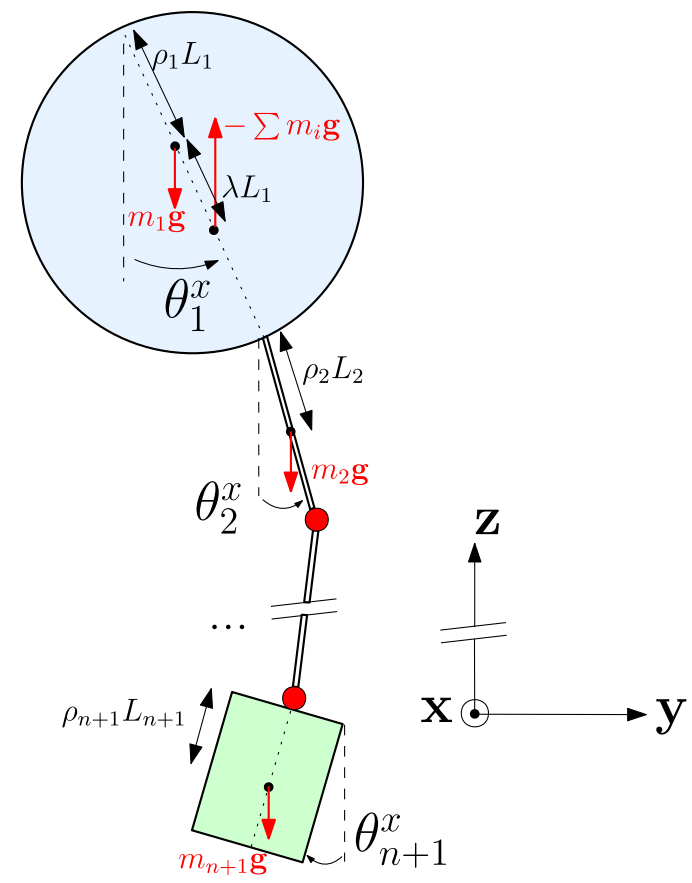

Fig. 1. Model of the pendulum dynamics of the system.

of interest for the pointing control, and (ii) the system experiences low vertical accelerations. In such operating conditions, all other aerodynamic forces and torques are considered as input disturbances. Small angles are assumed: the analysis of flight data shows that the gondola typically experiences angles not exceeding 0.2 degrees around the $\mathbf{x}$ and $\mathbf{y}$ axes, with periods going from the second (eigenmodes of the system) to hundreds of seconds (correlated with external disturbances) [3].

For $k>1$, the coordinates verify:

$$
\left\{\begin{array}{l}
y_{k}=y_{1}+\left(1-\rho_{1}\right) L_{1} \sin \theta_{1}^{x}+\sum_{i=2}^{k-1} L_{i} \sin \theta_{i}^{x}+\rho_{k} L_{k} \sin \theta_{k}^{x} \\
z_{k}=z_{1}-\left(1-\rho_{1}\right) L_{1} \cos \theta_{1}^{x}-\sum_{i=2}^{k-1} L_{i} \cos \theta_{i}^{x}-\rho_{k} L_{k} \cos \theta_{k}^{x} .
\end{array}\right.
$$

With the assumption of small motion, the terms in $\dot{\theta}_{k}^{x} \sin \left(\theta_{k}^{x}\right)$ are second order terms and can be neglected in the expression of the vertical velocities $\dot{z}_{k}$. Thus $\dot{z}_{k}=\dot{z}_{1}, \forall k$ and since the system is at an equilibrium altitude $\dot{z}_{k}=0$. The kinetic energy reads, with the assumption of small motion:

$2 E_{c}=\sum_{k=1}^{n+1}\left(m_{k} \dot{y}_{k}^{2}+I_{k}^{x}\left(\dot{\theta}_{k}^{x}\right)^{2}\right)$.

Let us choose the vector of $n+2$ degrees of freedom:

$\boldsymbol{\Theta}_{x}=\left[\begin{array}{llll}y_{1} & \theta_{1}^{x} & \ldots & \theta_{n+1}^{x}\end{array}\right]^{\mathrm{T}}$.

Let $\mu_{k}=\sum_{i=k}^{n+1} m_{i}$, and $\mu_{n+2}=0$. The potential energy derives from the buoyant force $-\mu_{1} \mathbf{g}$ applied to the center of pressure of the balloon, and from the weight $m_{k} \mathbf{g}$ of each body:

$E_{p}=-\mu_{1} g\left(z_{1}-\lambda L_{1} \cos \theta_{1}^{x}\right)+\sum_{k=1}^{n+1} m_{k} g z_{k}$

Under the small angle assumption, Lagrange's equations read:

$\mathbf{M}_{x} \ddot{\boldsymbol{\Theta}}_{x}+\mathbf{K}_{x} \boldsymbol{\Theta}_{x}=\mathbf{T}_{x}$ 


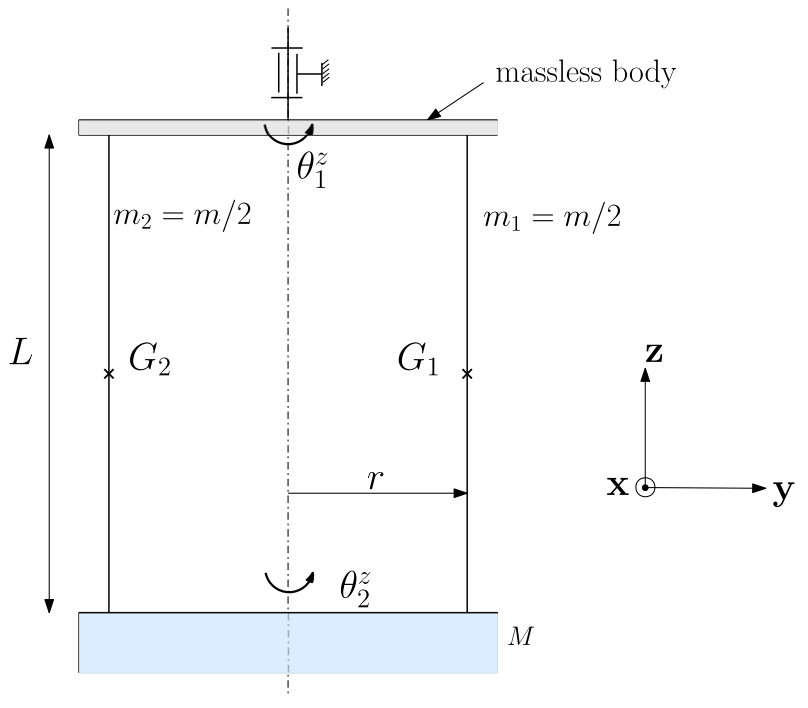

Fig. 2. Bifilar pendulum model.

where $\mathbf{T}_{x}$ is the vector of generalized force and torques. $\mathbf{M}_{x}$ and $\mathbf{K}_{x}$ are respectively the mass and stiffness matrices for the pendulum dynamics. Their expressions are detailed in Eqs. (A.1) and (A.2) in Appendix A.

Additionally, the gondola's matrix of inertia at its center of gravity may have cross terms coupling the azimuth axis $\mathbf{z}$ with $\mathbf{x}$, when the loading of the gondola is not symmetrical with respect to the $\mathbf{y}$-axis. Let $I_{n+1}^{z}$ be the moment of inertia of the gondola around $\mathbf{z}$, and $I_{n+1}^{x z}$ the cross-term coupling $\mathbf{x}$ and $\mathbf{z}$. The equations of motion become:

$$
\begin{aligned}
& {\left[\begin{array}{c|c}
\mathbf{M}_{x} & \mathbf{0}_{(n+1) \times 1} \\
I_{n+1}^{x z} \\
\hline \mathbf{0}_{1 \times(n+1)} I_{n+1}^{x z} & I_{n+1}^{z}
\end{array}\right]\left[\begin{array}{c}
\ddot{\Theta}_{x} \\
\hline \ddot{\theta}_{n+1}^{z}
\end{array}\right]} \\
& +\left[\begin{array}{c|c}
\mathbf{K}_{x} & \mathbf{0} \\
\hline \mathbf{0} & 0
\end{array}\right]\left[\frac{\boldsymbol{\Theta}_{x}}{\theta_{n+1}^{z}}\right]=\left[\frac{\mathbf{T}_{x}}{-T_{p}^{z}}\right] .
\end{aligned}
$$

The vector of generalized forces and torques on the right-hand side of Eq. (6) takes into account: (i) the aerodynamic force $F_{\text {aero }}^{y}$ and torque $T_{\text {aero }}^{x}$ applied to the balloon, (ii) the torque $T_{n+1}^{x}$ applied on the gondola by a reaction wheel to damp the pendulum motion around $\mathbf{x}$-axis and (iii) the reaction torque $-T_{p}^{z}$ applied by the azimuth driving mechanism in the motorized pivot between the gondola and the body $n$ :

$\left[\begin{array}{ll}\mathbf{T}_{x}^{T} & -T_{p}^{z}\end{array}\right]=\left[\begin{array}{lllll}F_{\text {aero }}^{y} & T_{\text {aero }}^{x} & \mathbf{0}_{1 \times(n-1)} & T_{n+1}^{x} & -T_{p}^{z}\end{array}\right]$.

\subsection{Torsion dynamics modeling}

The bifilar suspension, which is an essential element of many balloon-borne flight chains, is modeled with Lagrangian mechanics. The torsion dynamics of the flight chain are then derived.

\subsubsection{Bifilar suspension}

A sketch of a bifilar pendulum is shown in Fig. 2, constituted of two cables carrying a body of mass $M$ and moment of inertia $I$ around $\mathbf{z}$ at its center of gravity. Each cable has length $L$ and mass $m / 2$, and its center of gravity $G_{1}$ (resp. $G_{2}$ ) is in the middle of the cable. They are spaced by a distance $2 r$. The upper end of the cables cannot move along $\mathbf{z}$. Let $\theta_{1}^{z}$ be the angle around $\mathbf{z}$ of the top of the suspension, which is free to rotate around $\mathbf{z}$, and $\theta_{2}^{z}$ the angle around $\mathbf{z}$ of the bottom of the suspension, with regard to an inertial reference frame. The twist angle is $\eta=\theta_{2}^{z}-\theta_{1}^{z}$. At small angles, the torsion around $\mathbf{z}$ of an angle $\eta$ from the equilibrium position leads to a rotation of each cable around $\mathbf{y}$, in opposite directions, of angles $\theta^{y}= \pm \frac{r}{L} \eta$. In practice, the relative torsion angle does not exceed a few degrees during pointing operations, thus a linear approximation to small angles is justified.

The two cables bring the same contribution to the kinetic energy. Given a single cable of mass $m_{1}=m / 2$ and moment of inertia $J_{1}=\frac{m_{1} L^{2}}{12}$ around $\mathbf{y}$ at point $G_{1}$, and noting $\mathbf{V}_{G_{1}}$ its velocity vector at point $G_{1}$ and $\omega_{1}$ its angular velocity vector with regard to the inertial frame, its contribution to the kinetic energy reads at small angles:

$$
\begin{aligned}
2 E_{c 1} & =m_{1}\left\|\mathbf{V}_{G_{1}}\right\|^{2}+J_{1}\left\|\boldsymbol{\omega}_{1}\right\|^{2} \\
& =m_{1}\left[r \dot{\theta}_{1}^{z}+\frac{L}{2}\left(\frac{r}{L} \dot{\eta}\right)\right]^{2}+\frac{m_{1} L^{2}}{12}\left(\frac{r}{L} \dot{\eta}\right)^{2} \\
& =\frac{m_{1} r^{2}}{4}\left(\dot{\theta}_{1}^{z}+\dot{\theta}_{2}^{z}\right)^{2}+\frac{m_{1} r^{2}}{12}\left(\dot{\theta}_{2}^{z}-\dot{\theta}_{1}^{z}\right)^{2} .
\end{aligned}
$$

The contribution of the other cable is identical: $E_{c 2}=E_{c 1}$. The body of moment of inertia $I$ rotates of an angle $\theta_{2}^{z}$, its contribution $E_{c 3}$ to the kinetic energy verifies: $2 E_{c 3}=I\left(\dot{\theta}_{2}^{z}\right)^{2}$. Under the small angle assumption, the vertical velocity of the body of mass $M$ involves second order terms in $\dot{\eta} \eta$, and thus its translation kinetic energy can be neglected in $E_{c 3}$. Furthermore, let us note $I_{12}=m r^{2}$. The total kinetic energy $E_{c}=E_{c 1}+E_{c 2}+E_{c 3}$ reads:

$2 E_{c}=\frac{I_{12}}{4}\left(\dot{\theta}_{1}^{z}+\dot{\theta}_{2}^{z}\right)^{2}+\frac{I_{12}}{12}\left(\dot{\theta}_{2}^{z}-\dot{\theta}_{1}^{z}\right)^{2}+I\left(\dot{\theta}_{2}^{z}\right)^{2}$.

When the suspension is twisted with an angle $\eta$, the mass $M$ is elevated over an height $L\left[1-\cos \left(\frac{r}{L} \eta\right)\right]$. The center of gravity of each cable, of mass $\frac{m}{2}$, is elevated over an height $\frac{L}{2}\left[1-\cos \left(\frac{r}{L} \eta\right)\right]$. The potential energy reads:

$E_{p}=\left(M+\frac{m}{2}\right) g L\left[1-\cos \left(\frac{r}{L}\left(\theta_{2}^{z}-\theta_{1}^{z}\right)\right)\right]$.

\subsubsection{Torsion dynamics of the flight chain}

Same notations as for the pendulum motion are used: the bodies are numbered from 1 to $n+1$, where 1 is the balloon and $n+1$ is the gondola. In the proposed model, a body $k$ can either represent a rigid body, either a bifilar suspension. Both bring different contributions to the kinetic energy, and only the torsion of bifilar suspensions contributes to the potential energy. Each bifilar suspension $k$ is defined with its radius $r_{k}$, its length $L_{k}$, its mass $m_{k}$, and its moment of inertia $I_{k}^{z}=m_{k} r_{k}^{2}$. Each rigid body $k$ is defined with its mass $m_{k}$ and its moment of inertia $I_{k}^{z}$ at its center of gravity. Note that the bifilar suspension can represent an un-deployed parachute in the modeling of the torsion dynamics (see [17] for example). Without loss of generality, an alternation of rigid bodies and bifilar suspensions can be assumed. Indeed, a fictitious rigid body $k$ can be inserted between two successive bifilar suspensions, with $I_{k}^{z}=0$ and $m_{k}=0$. The bodies $1,3, \ldots, n-2, n$, are rigid bodies, and the bodies $2,4,6, \ldots, n-3, n-1$, are bifilar suspensions ( $n$ is thus an odd integer for the torsion model). Then, only the angles of the rigid bodies are defined since they are sufficient to describe the system.

When the body $k$ is a rigid body, its contribution $E_{c k}$ to the kinetic energy reads:

$2 E_{c k}=I_{k}^{z}\left(\dot{\theta}_{k}^{z}\right)^{2}$.

From the bifilar model Eq. (8), the contribution $E_{c k}$ of a bifilar suspension $k$ to the kinetic energy reads:

$2 E_{c k}=\frac{I_{k}^{z}}{4}\left(\dot{\theta}_{k+1}^{z}+\dot{\theta}_{k-1}^{z}\right)^{2}+\frac{I_{k}^{z}}{12}\left(\dot{\theta}_{k+1}^{z}-\dot{\theta}_{k-1}^{z}\right)^{2}$. 
Let $\mu_{k}=\sum_{i=k}^{n+1} m_{i}$, and $\mu_{n+2}=0$. The contribution $E_{p k}$ of a bifilar suspension $k$ to the potential energy reads (Eq. (10)):

$E_{p k}=\left(\frac{m_{k}}{2}+\mu_{k+1}\right) g L_{k}\left[1-\cos \left(\frac{r_{k}}{L_{k}}\left(\theta_{k+1}^{z}-\theta_{k-1}^{z}\right)\right)\right]$.

The degrees of freedom are the angles of the rigid bodies:

$\boldsymbol{\Theta}_{z}=\left[\begin{array}{lllll}\theta_{1}^{z} & \theta_{3}^{z} & \ldots & \theta_{n-2}^{z} & \theta_{n}^{z}\end{array}\right]^{T}$.

The degrees of freedom do not include the gondola's azimuth, since it was already accounted for in the pendulum dynamics (Eq. (6)) and is independent from the torsion of the flight chain in open-loop (the connection between the body $n$ and the gondola is a free pivot, in open-loop).

Under the small angles assumption, Lagrange's equations read:

$\mathbf{M}_{z} \ddot{\Theta}_{z}+\mathbf{K}_{z} \boldsymbol{\Theta}_{z}=\mathbf{T}_{z}$

$\mathbf{M}_{x}$ and $\mathbf{K}_{x}$ are respectively the mass and stiffness matrices for the torsion dynamics. Their expressions are detailed in Eqs. (A.3) and (A.4) in Appendix A. The vector of generalized torques $\mathbf{T}_{z}=\left[T_{\text {aero }}^{z} \mathbf{0}_{1 \times(n-3) / 2} T_{p}^{z}\right]^{T}$ takes into account (i) the aerodynamic torque $T_{z}^{\text {aero }}$ applied to the balloon and (ii) the control torque $T_{p}^{z}$ applied to the bottom of the flight chain by the motorized pivot.

\subsection{Coupled pendulum-torsion-azimuth model}

Finally, the complete dynamical model about the $\mathbf{x}$ and $\mathbf{z}$-axes reads:

$\mathbf{M} \ddot{\mathbf{X}}+\mathbf{K X}=\left[\begin{array}{lll}\mathbf{T}_{x}^{T} & \mathbf{T}_{z}^{T}-T_{p}^{z}\end{array}\right]^{T}$

With the vector of degrees of freedom:

$\mathbf{X}=\left[\begin{array}{lll}\boldsymbol{\Theta}_{x}^{T} & \boldsymbol{\Theta}_{z}^{T} & \theta_{n+1}^{z}\end{array}\right]^{T}$

and the symmetrical matrices $\mathbf{M}$ and $\mathbf{K}$ :

$\mathbf{M}=\left[\begin{array}{c|c|c}\mathbf{M}_{x} & \mathbf{0} & \begin{array}{c}\mathbf{0}_{(n+1) \times 1} \\ I_{n+1}^{x z}\end{array} \\ \hline \mathbf{0} & \mathbf{M}_{z} & \mathbf{0} \\ \hline \mathbf{0}_{1 \times(n+1)} I_{n+1}^{x z} & \mathbf{0} & I_{n+1}^{z}\end{array}\right]$

$\mathbf{K}=\operatorname{diag}\left(\mathbf{K}_{x}, \mathbf{K}_{z}, 0\right)$

In this paper, the pendulum dynamics about the $\mathbf{y}$-axis are assumed to be decoupled with the dynamics around $\mathbf{x}$ and $\mathbf{z}$ : $I_{n+1}^{x y}=I_{n+1}^{y z}=0$. They can be modeled similarly to those around $\mathbf{x}$.

The motorized pivot couples the torsion of the flight chain with the gondola's azimuth. This model also shows that the azimuth control torque $T_{p}^{z}$ can trigger pendulum oscillations as well because of the inertia coupling, expressed with the moment of inertia $I_{n+1}^{x z}$.

The natural damping of balloon-borne flight trains is generally low and difficult to estimate, because it originates from aerodynamic effects and from the friction in non-ideal joints. A damping matrix $\mathbf{C}$ can be introduced such that (16) is rewritten as:

$\mathbf{M} \ddot{\mathbf{X}}+\mathbf{C} \dot{\mathbf{X}}+\mathbf{K X}=\left[\begin{array}{lll}\mathbf{T}_{x}^{T} & \mathbf{T}_{z}^{T}-T_{p}^{z}\end{array}\right]^{T}$

Considering the modal matrix $\boldsymbol{\Phi}$, composed of the eigenvectors of the system, the modal damping matrix $\mathbf{C}_{m}=\boldsymbol{\Phi}^{T} C \boldsymbol{\Phi}$ is generally assumed to be diagonal. In this case, $\mathbf{C}_{m}$ is called a matrix of proportional damping [26, Chap. 2.2.2] and reads $\mathbf{C}_{\mathbf{m}}=\operatorname{diag}\left(2 \zeta_{i} \omega_{i}\right)$, where $\zeta_{i}$ and $\omega_{i}$ are respectively the damping and the frequency of the $i$ th mode. Typical modal damping values $\zeta_{i}$ are around $10^{-3}$.

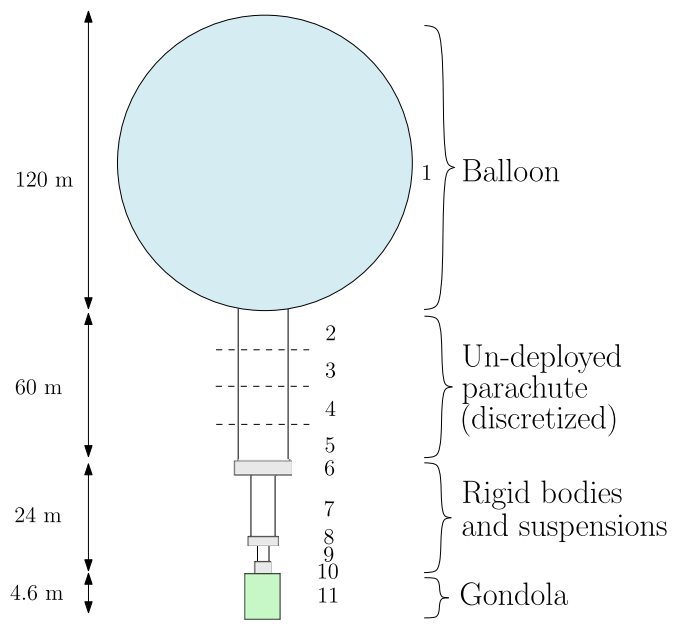

Fig. 3. Sketch of the FIREBall system (not in scale).

\subsection{Application of the modeling method and comparison with flight} data

In this section, the modeling approach is applied to the Faint Intergalactic-medium Redshifted Emission Balloon (FIREBall) experiment. The system consists of 11 bodies represented in Fig. 3. The values of the parameters are given in Table B.3 in Appendix B.

The main sources of system uncertainties are:

- the shape of the balloon, which can inflate and deflate throughout the flight and leads to uncertainties in the length, moment of inertia, position of the center of gravity, position of the center of buoyancy,

- the bifilar pendulum model, which might not capture all stiffness sources,

- the flexible dynamics of the un-deployed parachute, approximated by a discretization in 4 elements. Such a discretization leads to a 8-th order model for the parachute alone with 4 torsion modes. That was considered representative enough within the scope of this study,

- the mass and moment of inertia of the gondola, which depend on the ballast mass that was dropped during the flight,

- the static stretching of the parachute and bifilar suspension,

- the behavior of the connections between the bodies, idealized in the model.

Table 1 presents the frequencies of the eigenmodes predicted by the proposed model, i.e. the solutions in $\omega$ of the characteristic equation $\operatorname{det}\left(\mathbf{M} \omega^{2}-\mathbf{K}\right)=0$ where $\mathbf{M}$ and $\mathbf{K}$ are defined by equations (18) and (19), respectively.

The zero-frequency modes are not shown. The structural modes of the gondola are estimated greater than $1000 \mathrm{rad} \mathrm{s}^{-1}$ and are not considered in this study. Because of the coupling $I_{n+1}^{X z}$, the pendulum modes around $\mathbf{x}$-axis also affect the azimuth of the gondola. The pendulum modes around $\mathbf{y}$-axis are independent from the two other axes $\left(I_{n+1}^{x y}=I_{n+1}^{y z}=0\right)$, but they are modeled similarly with the multiple pendulum model.

The modes of Table 1 can be compared to the spectral analysis of the FIREBall 2018 flight [3]. The amplitude spectrum of the gondola's rotation rates around the three axes is presented in Fig. 4. The data were recorded over a period of 4 hours with a sampling time of $120 \mathrm{~ms}$ while the attitude control system was active. The altitude was approximately constant around $38 \mathrm{~km}$, and the gondola's angles did not exceed $0.2^{\circ}$ around $\mathbf{x}$ and $\mathbf{y}$, and $0.05^{\circ}$ around z. The 3-axes Inertial Measurement Unit (IMU) hybridizes the mea- 
Table 1

Eigenfrequencies (rad/s) predicted by the proposed model.

\begin{tabular}{llllllllllll}
\hline Mode & 1 & 2 & 3 & 4 & 5 & 6 & 7 & 8 & 9 & 10 & 11 \\
\hline Pendulum (x) & 0.265 & 0.831 & 2.53 & 3.47 & 6.45 & 11.6 & 18.1 & 20.3 & 44.2 & 63.9 & 128 \\
Pendulum (y) & 0.265 & 0.831 & 2.55 & 3.48 & 6.45 & 11.6 & 18.1 & 20.3 & 44.2 & 63.9 & 128 \\
Torsion $(\mathbf{z})$ & 1.81 & 3.94 & 6.42 & 7.03 & 11.9 & 18.3 & & & & & \\
\hline
\end{tabular}

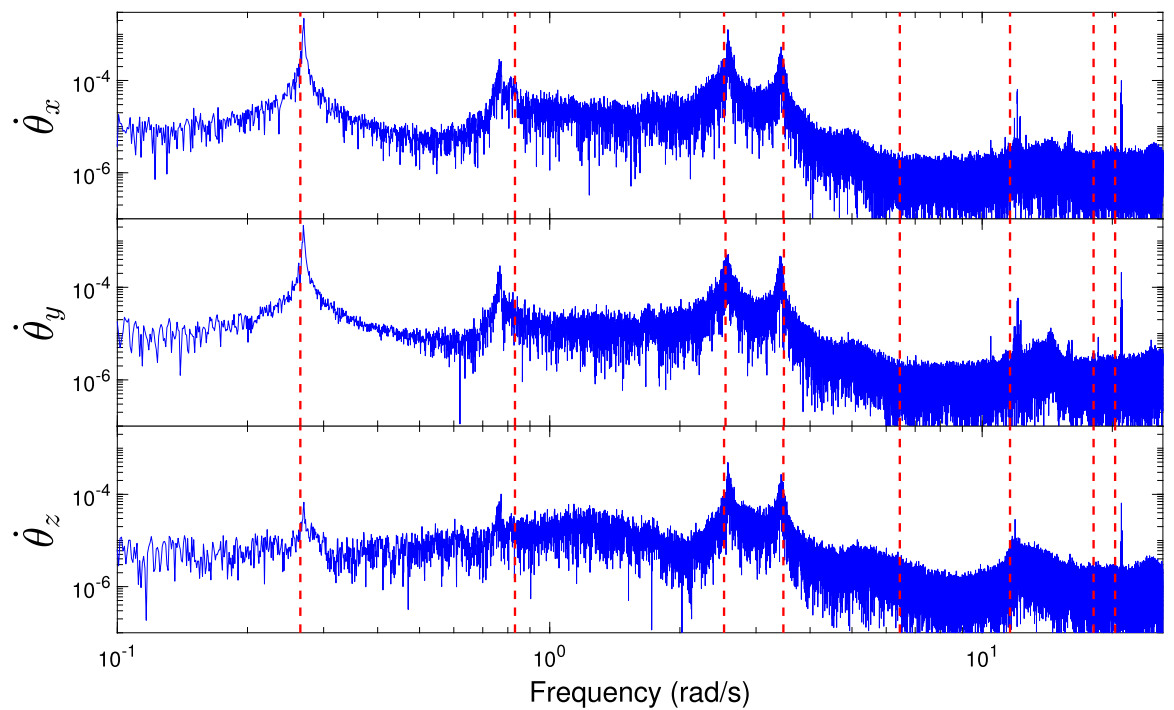

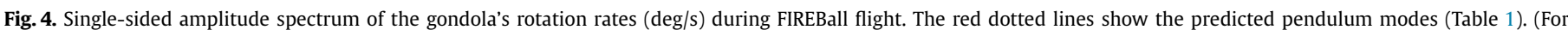
interpretation of the colors in the figure(s), the reader is referred to the web version of this article.)

Table 2

Relative error (in \%) between the model and the flight data.

\begin{tabular}{lllllllll}
\hline Mode & 1 & 2 & 3 & 4 & 5 & 6 & 7 & 8 \\
\hline Pendulum (x) & 1.8 & 8.7 & 2.1 & 1.3 & - & 3.6 & - & 3.2 \\
Pendulum (y) & 1.6 & 8.3 & 0.5 & 2.0 & - & 3.9 & - & 3.1 \\
\hline
\end{tabular}

surements of accelerometers with Fiber-Optic Gyroscopes (FOG) at $25 \mathrm{~Hz}$ sampling rate. The hardware used during FIREBAll flight is more detailed in [3,27].

The pendulum modes $1,2,3,4,6$ and 8 match resonance peaks observed on the spectra. The relative errors are presented in Table 2. However, we cannot conclude with certainty about pendulum modes 5 and 7 or any torsion mode, since they do not exhibit distinguishable peaks on the flight data. They might be more damped than the other modes (naturally or under the effect of the closed-loop control), or not excited by the disturbances and control. The modes $9,10,11$ are beyond the Nyquist frequency. It can be noticed that the pendulum modes are visible on $\dot{\theta}_{z}$ because of the inertia coupling that justified the proposed model.

The modal shapes of the pendulum modes 1 to 8 (around the $\mathbf{y}$ axis) are presented in Fig. 5. Mode 1 is an oscillation of the whole system around its center of gravity. Mode 2 is an in-phase oscillation of the balloon and the flight chain. As a consequence, the frequencies of modes 1 and 2 are very sensitive to the uncertain balloon parameters, which may explain the errors up to $8.7 \%$ on mode 2 . The excitation of modes 1 and 2 is primarily correlated with low-frequency altitude variations [28,29]. Mode 3 is primarily a pendulation of the gondola around its center of gravity. Mode 4 includes vibrations of the flight chain and of the gondola. The pendulum modes 3 and 4 represented a large contribution to the telescope's pointing error during the FIREBall 2018 flight [3]. Modes 3 and 4 can be excited by wind gusts [18], but also by the azimuth control of the gondola because of the inertia coupling. Pendulum modes of higher frequency ( 5 and more) are essentially oscillations of the elements of the flight train.

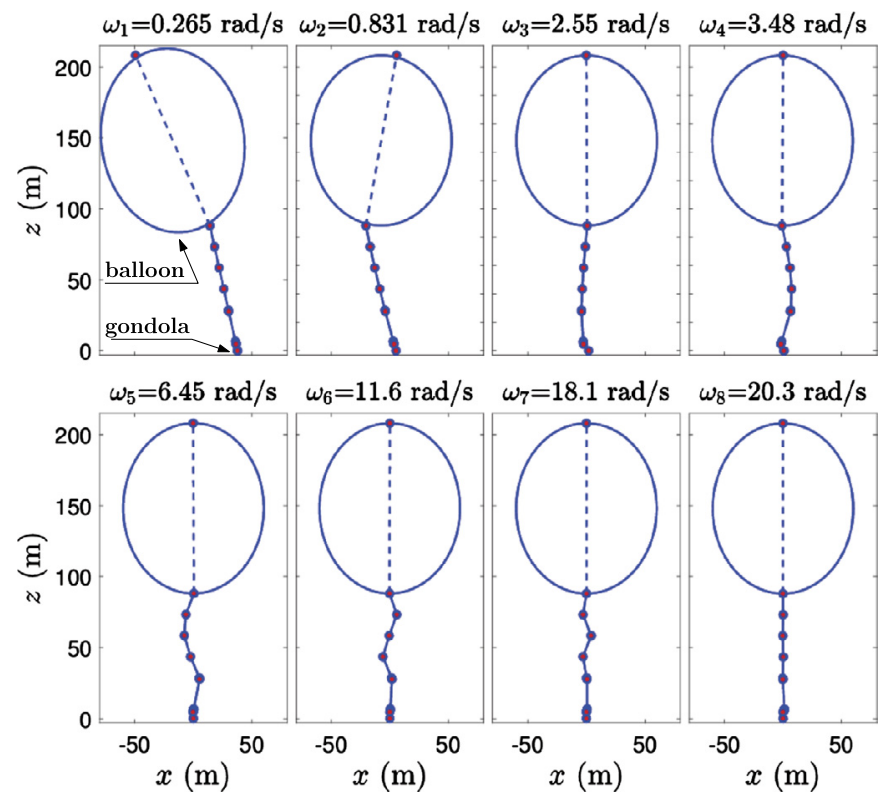

Fig. 5. In scale modal shapes of pendulum modes 1 to 8 in the $(\mathbf{x}, \mathbf{z})$ plane.

\section{Stability}

The azimuth pointing control of balloon-borne gondolas is most often designed solely with a model of the torsion of the flight train [7-15]. However, in presence of a coupling between the gondola's azimuth and the pendulum oscillations of the system, the azimuth control can excite and even destabilize the pendulum modes. The stability of the coupled pendulum-torsion-azimuth model proposed in Section 2 is investigated using the positivity framework. 

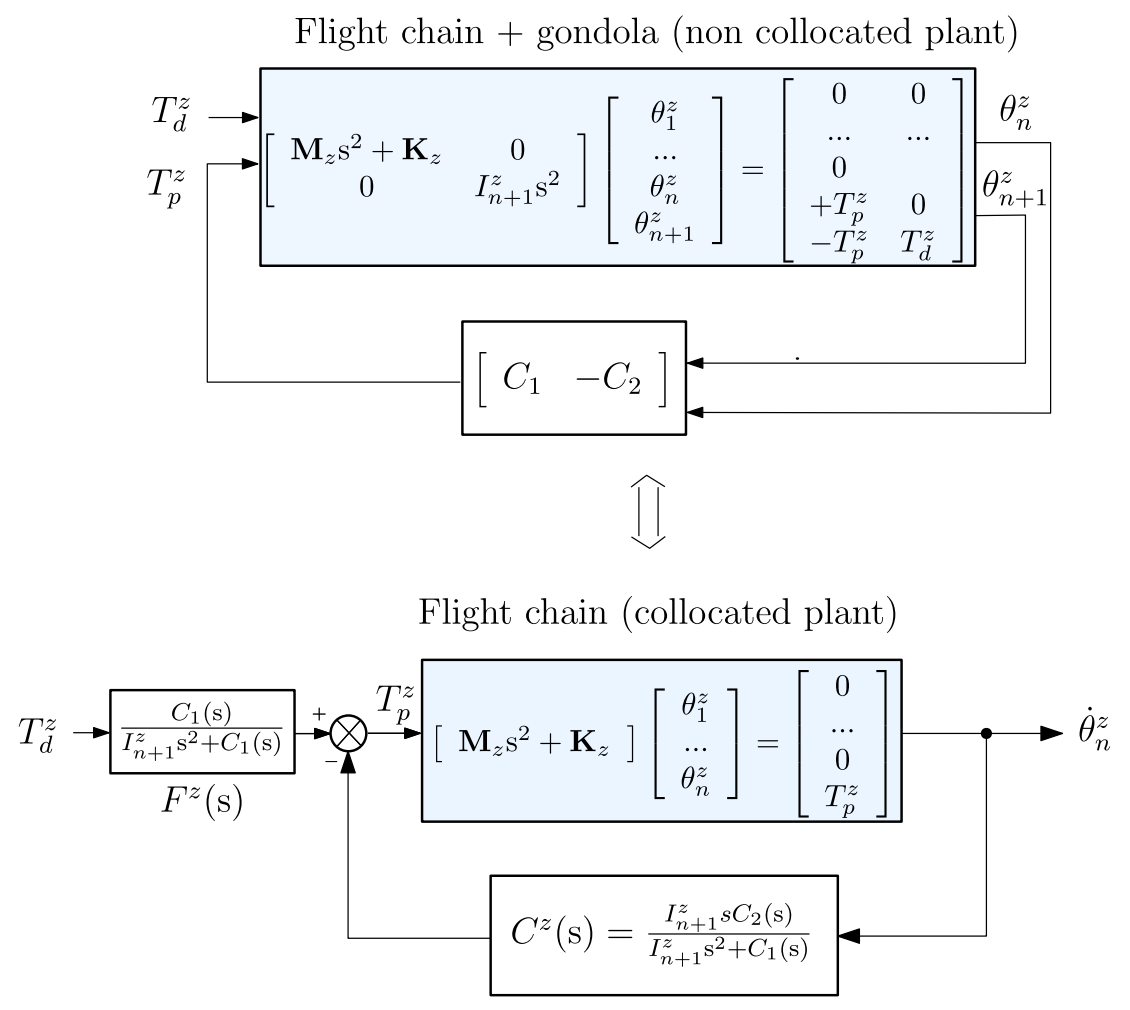

Fig. 6. Equivalence between the system and a collocated plant with a strictly positive real feedback. We note $Z$ (s) is the closed-loop transfer from $T_{d}^{z}$ to $\dot{\theta}_{n}^{z}$.

\subsection{The positivity framework}

The definitions and results from [24,25] are recalled here to introduce the positivity framework. This framework provides strong stability guarantees when using collocated actuators and sensors, and therefore it is widely used for the control of flexible structures [30].

Definition 1 (Positive realness [25]). A square transfer matrix $\mathbf{Z}(\mathrm{s}$ ), where $s$ is the Laplace variable, is positive real if it verifies: (i) $\mathbf{Z}$ (s) has real elements for real s, (ii) $\mathbf{Z}$ (s) has elements which are analytic for $\operatorname{Re}(\mathrm{s})>0$, and (iii) $\mathbf{Z}(\mathrm{s})+\mathbf{Z}^{*}(\mathrm{~s})$ is nonnegative definite for $\operatorname{Re}(\mathrm{s})>0\left(\mathbf{Z}^{*}(\mathrm{~s})\right.$ is the transpose conjugate of $\left.\mathbf{Z}(\mathrm{s})\right)$.

Definition 2 (Strict positive realness [25]). A square transfer matrix $\mathbf{Z}(\mathrm{s})$ is strictly positive real if it verifies: (i) $\mathbf{Z}(\mathrm{s})$ has real elements for real $s$, (ii) $\mathbf{Z}(\mathrm{s})$ has elements which are analytic for $\operatorname{Re}(\mathrm{s}) \geq 0$, and (iii) $\mathbf{Z}(j \omega)+\mathbf{Z}^{*}(j \omega)$ is positive definite for all real $\omega$.

Theorem 1 (Feedback of positive real systems [25]). If two systems $S_{1}$ and $S_{2}$ are put in feedback, and one of them is strictly positive real, and the other one is positive real, then the closed-loop system is asymptotically stable in the input-output sense. It also holds for all observable/controllable modes.

Theorem 2 (Collocated control [25]). The transfer matrix of a structure is positive real if collocated force (respectively torque) actuators and translation (respectively rotation) rate sensors are used. An actuator and a sensor are collocated if they are attached to the same degree of freedom.

\subsection{Stability of the torsion-azimuth model}

In this section, the matrix of inertia of the gondola is considered diagonal: $I_{n+1}^{x z}=0$. In this case, the torsion and pendulum dynamics are decoupled, even in closed-loop. A sufficient condition on the azimuth controller gains is derived to ensure the asymptotic stability of the closed-loop torsion-azimuth model.

The azimuth control presented here relies on one actuator (the motorized pivot), which applies a torque $+T_{p}^{z}$ to the bottom of the flight chain and $-T_{p}^{z}$ to the gondola, and two sensors: the gondola's rotation rate $\dot{\theta}_{n+1}^{z}$, and the relative rotation rate $\dot{\theta}_{n}^{z}-\dot{\theta}_{n+1}^{z}$ measured by the instrumentation of the pivot. A disturbance torque $T_{d}^{z}$ is applied to the gondola around $\mathbf{z}$. Both actuator and sensor dynamics are considered high enough in frequency bandwidth to be neglected in the positivity analysis.

The torsion model (15) augmented with the gondola's azimuth dynamic model:

$-T_{p}^{z}+T_{d}^{z}=I_{n+1}^{z} \mathrm{~s}^{2} \theta_{n+1}^{z}$,

in closed loop with a general control law under the form:

$T_{p}^{z}=C_{1}(\mathrm{~s}) \theta_{n+1}^{z}-C_{2}(\mathrm{~s}) \theta_{n}^{z}$

is represented in Fig. 6 (top block-diagram). $C_{1}(\mathrm{~s})$ and $C_{2}(\mathrm{~s})$ are dynamic controllers.

However, this architecture involves a non-square model and a non-square controller. Thus, the positivity and colocation framework does not allow to conclude on the closed-loop stability. Nevertheless, the closed-loop system can be written in an equivalent plant - feedback control interconnection, such that the new plant and controller are collocated. Indeed, by eliminating $\theta_{n+1}^{z}$ in equations (21) and (22), the closed-loop system can be interpreted as the bottom block diagram of Fig. 6. In this representation, (i) the plant from $T_{z}^{p}$ to $\dot{\theta}_{n}^{z}$ is collocated, thus positive real (Theorem 2), and (ii) $C^{z}$ (s) is an equivalent control whose the strict positive realness allows to characterize the asymptotic stability of the closedloop system (Theorem 1).

From Definition 1 applied to the Single-Input Single-Output transfer function $C^{z}(s)$, the closed-loop asymptotic stability conditions can be stated. 
Condition 1 (Uncoupled azimuth stability).

- $C^{z}(\mathrm{~s})$ has real elements for real values of $\mathrm{s}$,

- the roots of $I_{n+1}^{z} \mathrm{~s}^{2}+C_{1}(\mathrm{~s})$ only have strictly negative real parts,

- $\operatorname{Re}\left(C^{z}(j \omega)\right)>0$ for all $\omega \in \mathbb{R}$.

When these conditions are met, since the plant is positive real, Theorem 1 states that all controllable modes of the closed-loop system are asymptotically stable.

\section{Remarks 1.}

- These conditions do not depend on the parameters of the flexible system. In particular, the knowledge of the eigenfrequencies is not even required to ensure asymptotic stability, and the result is valid for all flexible modes of any flight chain,

- they ensure also the stability of the equivalent feed-forward controller $F^{z}(\mathrm{~s})$, required for the stability of the initial-control interconnection.

- in the case of a pure collocated control $C_{1}(\mathrm{~s})=C_{2}(\mathrm{~s})$, the strict positive realness of $C_{2}(\mathrm{~s})$ ensures the asymptotic stability of the closed-loop system. But the pure collocated control cannot be applied during the pointing phase since the inertial attitude of the gondola must be controlled and regulated around 0 . Indeed the closed-loop steady state must ensures that $T_{p}^{z}=0$ and $\theta_{n+1}^{z}=\theta_{n}^{z}=0$ and not only $T_{p}^{z}=0$ and $\theta_{n+1}^{z}=\theta_{n}^{z}$ (from Eq. (22)). Thus $C_{1}(s) \neq C_{2}(s)$ and the stability property of the pure collocated control cannot be applied.

\subsection{Stability of the coupled pendulum-torsion-azimuth model}

When the matrix of inertia of the gondola has a non diagonal term coupling the azimuth axis with the roll axis: $I_{n+1}^{x z} \neq 0$, the Conditions 1 are no longer sufficient since the block-diagram equivalence presented in Fig. 6 is no longer valid. Indeed, in some configurations, the azimuth control might destabilize the pendulum oscillations, as it was suspected during previous Carmen (2015) and PILOT (2015 and 2017) flights. Given an azimuth controller ensuring the asymptotic stability of the torsion-azimuth model (considered independent from the pendulum dynamics), as addressed in Section 3.2, it is demonstrated that a reaction wheel placed on the $\mathbf{x}$-axis allows to ensure the asymptotic stability of the complete system.

The complete model (16) is considered with an azimuth controller (22), and a reaction wheel providing a torque $T_{n+1}^{x}=$ $-K(\mathrm{~s}) \dot{\theta}_{n+1}^{x}$ to damp the pendulum motion of the gondola, where $K(\mathrm{~s})$ is a dynamic feedback containing the wheel dynamics. The closed-loop system can be interpreted as the block diagram in Fig. 7, considering the coupling terms $I_{n+1}^{x z} \ddot{\theta}_{n+1}^{z}$ and $I_{n+1}^{x z} \ddot{\theta}_{n+1}^{x}$ as disturbance torques in, respectively, the pendulum model (top block) and the torsion model augmented with the gondola's azimuth (bottom block). $Z$ (s) is the transfer function from a disturbance torque $T_{d}^{z}$ applied to the gondola around $\mathbf{z}$, to the gondola's rotation rate $\dot{\theta}_{n+1}^{z}$ around $\mathbf{z}$, in the closed-loop torsion-azimuth model addressed in Fig. 6 (left block-diagram).

In the block diagram represented in Fig. 7, the plant from $T$ to $\dot{\theta}_{n+1}^{x}$ is collocated, and the equivalent negative feedback reads:

$C(s)=K(s)-\left(I_{n+1}^{x z} s\right)^{2} Z(s)$

and highlights that the torsion-azimuth closed-loop system $Z$ (s) may act as a destabilizing feedback for the pendulum system. However, the strict positive realness of $C(s)$ is sufficient to ensure the asymptotic stability of the system. It is assumed that the azimuth controller (22) is designed such that the strict

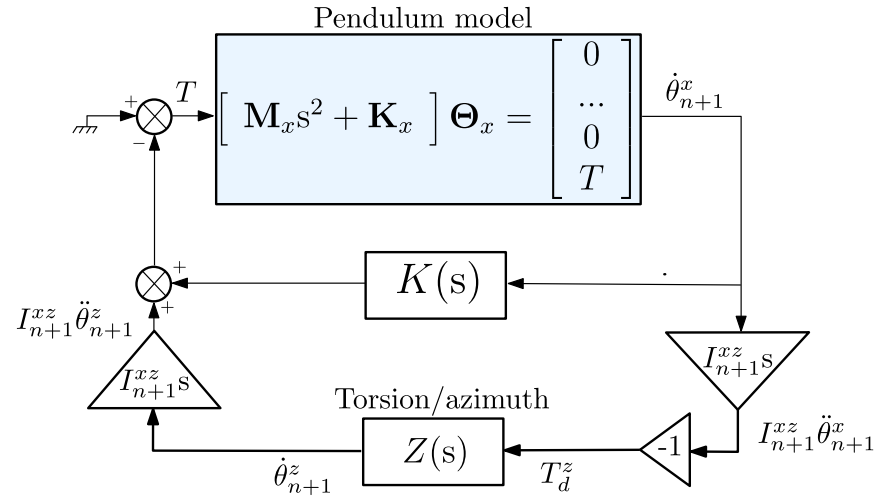

Fig. 7. Model with coupled pendulum and torsion/azimuth dynamics.

positive realness of $C^{z}(\mathrm{~s})$ is verified in Section 3.2. As a consequence, $Z(\mathrm{~s})$ is real when $\mathrm{s}$ is real, and the poles of $Z(\mathrm{~s})$ only have strictly negative real parts. It is immediate that $C(\mathrm{~s})$ also verifies these two properties if $K(\mathrm{~s})$ is also stable. Then, items (i) and (ii) are fulfilled in Definition 2, and the SingleInput Single-Output transfer function $C(s)$ is strictly positive real if and only if $\operatorname{Re}(C(j \omega))>0$ for all $\omega \in \mathbb{R}$ (item (iii)), thus:

Condition 2 (Coupled pendulum-azimuth stability).

$K(j \omega)+\left(I_{n+1}^{x z}\right)^{2} \omega^{2} \operatorname{Re}(Z(j \omega))>0, \quad \forall \omega \in \mathbb{R}$.

In the general case $I_{n+1}^{x z} \neq 0$, the stability is not guaranteed with the positivity framework when the motorized pivot is the only actuator $(K=0)$, since $Z(j \omega)$ can have negative values. The fully parameterized pendulum-torsion model (Eq. (20)) and the stability condition (Condition 2) can be used during preliminary design phases to size a flight chain and the associated control system. That is illustrated in the section 3.4.

\subsection{Illustration: destabilization of a pendulum mode by the azimuth control}

In this section, the risk of destabilizing the pendulum modes with an azimuth controller is illustrated with a numerical example. The design of the FIREBall system presented in Section 2.4 is such that this risk is very small. However, it was discussed to increase the length of the bifilar suspension (body 7 in Table B.3, in Appendix B) from $21 \mathrm{~m}$ to $40 \mathrm{~m}$ to reduce the exposure of the instrument to parasite light coming from the balloon by reflection. In this new configuration, the torsion mode 2 and the pendulum mode 4 have the same frequency in open-loop. Using the coupled pendulum-torsion-azimuth model proposed in Section 2, it is shown that the azimuth controller can destabilize the pendulum mode 4.

A proportional-derivative azimuth controller (Eq. (22)) is considered with $C_{i}(s)=K_{p i}+K_{d i} s, i=1,2$ and:

$$
\begin{array}{ll}
K_{p 1}=3330 \mathrm{~N} \mathrm{mrad}^{-1} & K_{d 1}=4440 \mathrm{~N} \mathrm{~ms} \mathrm{rad}^{-1} \\
K_{p 2}=23.3 \mathrm{~N} \mathrm{mrad}^{-1} & K_{d 2}=3.1 \mathrm{~N} \mathrm{~ms} \mathrm{rad}^{-1}
\end{array}
$$

This controller was designed solely on the nominal torsion model so as to provide a bandwidth of $0.7 \mathrm{rad} \mathrm{s}^{-1}$ while damping the torsion modes 1 and 2. 


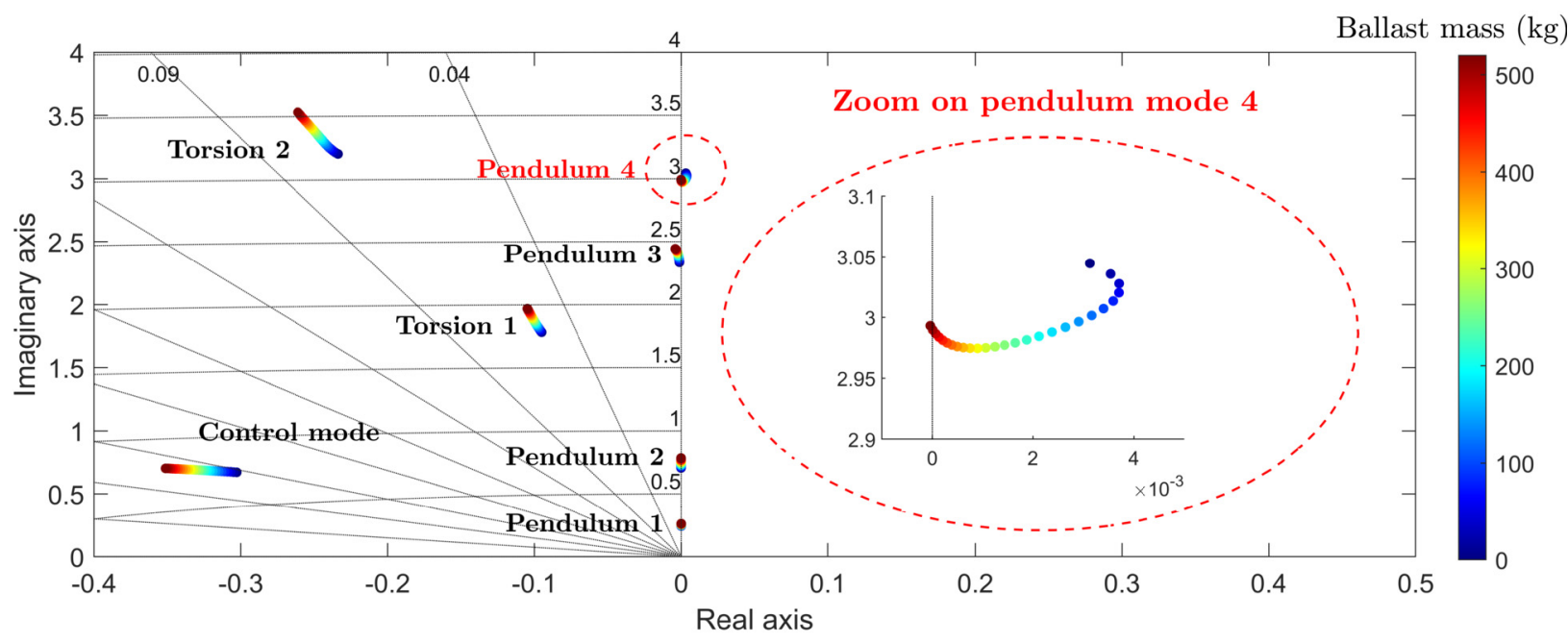

Fig. 8. Map of the closed-loop poles, for $K=0$ and varying ballast mass.

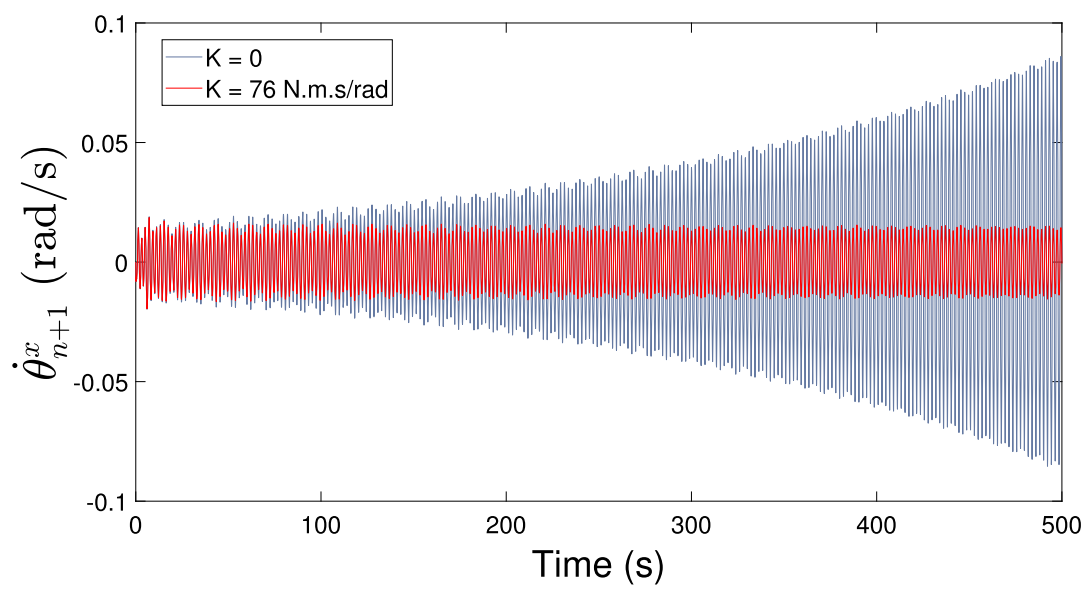

Fig. 9. Response to an initial azimuth error of $1 \mathrm{rad}$, for a ballast mass of $50 \mathrm{~kg}$.

The root locus of the system is presented in Fig. 8 with varying ballast mass, so as to represent all flight configurations. Only the first modes are represented for readability:

- azimuth control mode (rigid mode in open-loop, which was placed in closed-loop at a chosen dynamic of $0.7 \operatorname{rad~s}^{-1}$ ),

- torsion modes 1 and 2,

- pendulum modes 1 to 4 (near the imaginary axis).

The control mode and torsion modes are stable. However, as the ballast mass decays, the pendulum mode 4 becomes unstable. Nevertheless, an evaluation of $Z(j \omega)$ in condition (24) indicates that $K(s)=76 \mathrm{~N} \mathrm{~ms} \mathrm{rad}^{-1}$ (we neglect the wheel's dynamics) is sufficient to ensure condition (24), and thus the asymptotic stability of the system. This is illustrated with a simulation presented in Fig. 9 showing the response of the gondola's rotation rate $\dot{\theta}_{n+1}^{x}$ to an initial unitary pointing error in azimuth, for $K=0$ and $K=76 \mathrm{Nmsrad}^{-1}$.

\section{Conclusion}

A new fully parameterized model of balloon-borne flight trains was developed to address the coupling of the pendulum dynamics with the gondola's azimuth. The method was applied to the Faint Intergalactic-medium Redshifted Emission Balloon (FIREBall) experiment, and the modes predicted by the proposed model matched the resonance peaks on the spectral analysis of flight data. The stability of the proposed coupled pendulum-torsionazimuth model was investigated with the positivity framework and sufficient stability conditions were derived. It was shown how an azimuth control can destabilize the pendulum modes, and how the stability can be recovered with an additional actuator. Although the pointing performance was beyond the scope of this paper, previous flight experiences showed that the pendulum dynamics and their coupling with the gondola's azimuth largely contribute to the pointing error budget and thus motivated this work. The proposed model and stability conditions are general to any platforms and then can be used during preliminary design phases of new missions.

\section{Declaration of competing interest}

The authors declare that they have no known competing financial interests or personal relationships that could have appeared to influence the work reported in this paper.

\section{Funding sources}

This work was funded by ISAE-Supaero and CNES (French space agency - grant number: 51/18660). 
Table B.3

Parameters of the FIREBALL system.

\begin{tabular}{llllllll}
\hline Body & $m(\mathrm{~kg})$ & $L(\mathrm{~m})$ & $\rho(-)$ & $r(\mathrm{~m})$ & $I^{x}\left(\mathrm{~kg} \mathrm{~m}^{2}\right)$ & $I^{y}\left(\mathrm{~kg} \mathrm{~m}^{2}\right)$ & $I^{z}\left(\mathrm{~kg} \mathrm{~m}^{2}\right)$ \\
\hline 1 & 3231 & 120 & 0.32 & - & $5.12 \times 10^{6}$ & $5.12 \times 10^{6}$ & $5.12 \times 10^{6}$ \\
2 to 5 & 40.30 & 14.87 & 0.5 & 0.457 & 742 & 742 & 8.42 \\
6 & 54.93 & 1.00 & 0.463 & - & 6.51 & 6.51 & 8.85 \\
7 & 40.52 & 21 & 0.5 & 0.20 & 1489 & 1489 & 1.62 \\
8 & 16.5 & 0.45 & 0.5 & - & 0.278 & 0.278 & 2.06 \\
9 & 5.92 & 0.95 & 0.5 & 0.025 & 0.445 & 0.445 & 0.0037 \\
10 & 33.5 & 0.70 & 0.5 & - & 1.37 & 1.37 & 0.377 \\
11 & 2277 & 4.6 & 0.605 & - & 8510 & 8153 & 1538 \\
\hline
\end{tabular}

\section{Appendix A. Expression of the mass and stiffness matrices}

The matrices of the pendulum dynamics (5) read

$$
\begin{gathered}
\mathbf{M}_{x}=\left[\begin{array}{ccc}
\mu_{1} & \left(1-\rho_{1}\right) L_{1} \mu_{2} & \left(\rho_{2} m_{2}+\mu_{3}\right) L_{2} \\
* & I_{1}^{x}+\left(1-\rho_{1}\right)^{2} L_{1}^{2} \mu_{2} & \left(1-\rho_{1}\right)\left(\rho_{2} m_{2}+\mu_{3}\right) L_{1} L_{2} \\
* & * & I_{2}^{x}+\left(\rho_{2}^{2} m_{2}+\mu_{3}\right) L_{2}^{2} \\
\ldots & \ldots & \cdots \\
* & * & * \\
\ldots & \left(\rho_{n+1} m_{n+1}+\mu_{n+2}\right) L_{n+1} \\
\ldots & \left(1-\rho_{1}\right)\left(\rho_{n+1} m_{n+1}+\mu_{n+2}\right) L_{1} L_{n+1} \\
\ldots & \left(\rho_{n+1} m_{n+1}+\mu_{n+2}\right) L_{2} L_{n+1} \\
\ldots & \ldots
\end{array}\right] \\
\ldots \\
\mathbf{K}_{x}=\operatorname{diag}\left(0,\left(1-\rho_{1}\right) g L_{1} \mu_{2}-\lambda g L_{1} \mu_{1},\left(\rho_{i} m_{i}+\mu_{i+1}\right) g L_{i}\right),
\end{gathered}
$$

The matrices of the torsion dynamics (15) read

$$
\mathbf{M}_{z}=\left[\begin{array}{ccccc}
I_{1}^{z}+\frac{I_{2}^{z}}{3} & \frac{I_{2}^{z}}{6} & \ldots & 0 & 0 \\
\frac{I_{2}^{z}}{6} & \frac{I_{2}^{z}}{3}+I_{3}^{z}+\frac{I_{4}^{z}}{3} & \ldots & 0 & 0 \\
\ldots & \ldots & \ldots & \ldots & \ldots \\
0 & 0 & \ldots & \frac{I_{n-3}^{z}}{3}+I_{n-2}^{z}+\frac{I_{n-1}^{z}}{3} & \frac{I_{n-1}^{z}}{6} \\
0 & 0 & \ldots & \frac{I_{n-1}^{z}}{6} & \frac{I_{n-1}^{z}}{3}+I_{n}^{z}
\end{array}\right]
$$

$\mathbf{K}_{z}=\left[\begin{array}{cccccc}K_{2} & -K_{2} & \ldots & 0 & 0 & \\ -K_{2} & K_{2}+K_{4} & \ldots & 0 & 0 & \\ \ldots & \ldots & \ldots & \ldots & \ldots & \ldots \\ 0 & 0 & \ldots & K_{n-3}+K_{n-1} & -K_{n-1} & \\ 0 & 0 & \ldots & -K_{n-1} & K_{n-1} & \end{array}\right]$

with $K_{k}=\left(\frac{m_{k}}{2}+\mu_{k+1}\right) \frac{g r_{k}^{2}}{L_{k}} \quad$ when the body $k$ is a bifilar

suspension.

\section{Appendix B. Model data}

The model discussed in Section 2.4 uses the data given in Table B.3, and the values $\mathrm{g}=9.70 \mathrm{~m} / \mathrm{s}^{2}, \lambda=0.1$, and $I_{n+1}^{X z}=-467$ $\mathrm{kg} \mathrm{m}^{2}$. Body 1 is the balloon. Bodies 2 to 5 represent the undeployed parachute, which is discretized in 4 elements to capture its flexibility. Bodies 6 to 10 are other suspensions or rigid bodies of the flight chain. Body 11 is the gondola equipped with ballast. The ballast mass affects the mass of the gondola, the position of its center of gravity and its inertia. In this model, a ballast mass of $400 \mathrm{~kg}$ is considered. When the radius $r$ is specified, the element is modeled as a bifilar suspension. Otherwise, it is a rigid body.

\section{References}

[1] N. Yajima, T. Imamura, N. Izutsu, T. Abe, Scientific Ballooning: Technology and Applications of Exploration Balloons Floating in the Stratosphere and the Atmospheres of Other Planets, Springer Science \& Business Media, New-York, 2009.

[2] W.V. Jones, Evolution of scientific ballooning and its impact on astrophysics research, Adv. Space Res. 53 (2014) 1405-1414.

[3] J. Montel, E. Pérot, F. Mirc, J. Evrard, N. Melso, D. Schiminovich, FIREBALL-2 (2018) in-flight pointing performance, in: 24th ESA Symposium on European Rocket and Balloon Programmes and Related Research, ESA Publications, Essen, Germany, 2019, pp. 51-57.

[4] D.W. Stuchlik, The wallops arc second pointer - a balloon borne fine pointing system, in: AIAA Balloon Systems Conference 2015, MBAL 2015, 2015 pp. 1-15, held at the AIAA Aviation Forum 2015.

[5] G.A. Howe, C.B. Mendillo, K. Hewawasam, S. Chakrabarti, T.A. Cook, J. Martel, S.C. Finn, The low-order wavefront control system for the PICTURE-C mission: preliminary testbed results from the Shack-Hartmann sensor, in: Proceedings of SPIE - The International Society for Optical Engineering, 2017, p. 70.

[6] L.J. Romualdez, S.J. Benton, A.M. Brown, P. Clark, C.J. Damaren, T. Eifler, A.A. Fraisse, M.N. Galloway, A. Gill, J.W. Hartley, B. Holder, E.M. Huff, M. Jauzac, W.C. Jones, D. Lagattuta, J.S. Leung, L. Li, T.V.T. Luu, R.J. Massey, J. McCleary, J. Mullaney, J.M. Nagy, C.B. Netterfield, S. Redmond, J.D. Rhodes, J. Schmoll, M.M. Shaaban, E. Sirks, S.I. Tam, Robust diffraction-limited NIR-to-NUV wide-field imaging from stratospheric balloon-borne platforms-SuperBIT science telescope commissioning flight \& performance, 2019, pp. 1-14, arXiv.

[7] J.E. White, J.R. Etter, Linear-quadratic-regulator pointing control system for a high-altitude balloon payload, J. Guid. Control Dyn. 13 (1990) 615-623.

[8] A.O. Chingcuanco, P.M. Lubin, P.R. Meinhold, M. Tomizuka, Modeling and control of a balloon borne stabilized platform, J. Dyn. Syst. Meas. Control 112 (1990) 703-710

[9] A.O. Chingcuanco, P.M. Lubin, P.R. Meinhold, M. Tomizuka, Model reference adaptive control for the azimuth-pointing system of a balloon-borne stabilized platform, Int. J. Adapt. Control Signal Process. 5 (1991) 107-120.

[10] D. Matko, N. Yajima, M. Hinada, Adaptive stabilisation of lateral motion of a balloon gondola, Control Eng. Pract. 5 (1997) 763-769.

[11] P.R. Ward, K.D. DeWeese, Balloon borne arcsecond pointer feasibility study, in: 16th ESA Symposium on European Rocket and Balloon Programmes and Related Research, vol. 530, ESA Publications, St. Gallen, Switzerland, 2003, pp. 197-205.

[12] Y. Sakamoto, T. Kanazawa, Y. Shouji, Y. Takahashi, K. Yoshida, M. Taguchi, Dynamic modeling and experimental verification of the pointing technology in balloon-borne telescope system for optical remote sensing of planets, Trans. Jpn. Soc. Aeronaut. Space Sci., Space Technol. Jpn. 7 (2009) 23-28.

[13] T. Nakano, Y. Shoji, M. Yamamoto, K. Hamamoto, J. Nakamoto, M. Imai, Y Sakamoto, T. Kuwahara, M. Watanabe, Y. Takahashi, K. Yoshida, M. Taguchi, The pointing control method of balloon-borne telescope compensating the motion of flexible base, in: IEEE/SICE International Symposium on System Integration, IEEE, Fukuoka, Japan, 2012, pp. 313-318.

[14] T. Nakano, Design of attitude control system for stratospheric balloon gondolas by sliding mode control, in: Conference on Mechanical Engineering, Phuket, Thailand, in: IOP Conference Series: Materials Science and Engineering, vol. 501, 2019

[15] D. Zhang, J. Zhou, W. Huang, Design of decoupling control system for the azimuth control of balloon-borne mission, in: 35th Chinese Control Conference, IEEE, Chengdu, China, 2016.

[16] L.M. Fissel, PHD Thesis - Probing the Role Played by Magnetic Fields in Star Formation with BLASTPol, Ph.D. thesis, 2013.

[17] F. Aubin, B. Bayman, S. Hanany, H. Franco, J. Marsh, J. Didier, A.D. Miller, Torsional balloon flight line oscillations: comparison of modelling to flight data, Adv. Space Res. 60 (2017) 702-708.

[18] N.J. Nigro, J.K. Yang, A.F. Elkouh, N.J. Nigro, Generalized math model for simulation of high-altitude balloon systems, J. Aircr. 22 (1985) 697-704.

[19] M.B. Quadrelli, J.M. Cameron, V. Kerzhanovich, Multibody dynamics of parachute and balloon flight systems for planetary exploration, J. Guid. Control Dyn. 27 (2004) 564-571. 
[20] L.J. Romualdez, Design, Implementation, and Operational Methodologies for Sub-Arcsecond Attitude Determination, Control, and Stabilization of the Superpressure Balloon-borne Imaging Telescope (SuperBIT), Ph.D. thesis, 2018.

[21] T. Nakano, M. Taguchi, Y. Shoji, M. Takamura, D. Sunaguchi, M. Imai, M. Watanabe, Y. Takahashi, Y. Sakamoto, K. Yoshida, Characteristics evaluation and performance improvement method of balloon-borne telescope pointing control system, Trans. Jpn. Soc. Aeronaut. Space Sci. 17 (2019) 51-56.

[22] I.S. Smith, E.A. Chaffee, J.R. Noll, B.A. Martin, The investigation and measurement of balloon dynamics at the apex and base of a scientific balloon, in: AIAA Balloon Systems Conference, AIAA, Denver, CO, 2017, p. 3286.

[23] E. Hamden, D.C. Martin, B. Milliard, D. Schiminovich, S. Nikzad, J. Evrard, G, Kyne, R. Grange, J. Montel, E. Pirot, K. Hoadley, D. O'sullivan, N. Melso, V. Picouet, D. Vibert, P. Balard, P. Blanchard, M. Crabill, S. Pascal, F. Mirc, N. Bray, A. Jewell, J. Blue Bird, J. Zorilla, H.R. Ong, M. Matuszewski, N. Lingner, R. Augustin, M. Limon, A. Gomes, P. Tapie, X. Soors, I. Zenone, M. Saccoccio, FIREBall-2: the faint intergalactic medium redshifted emission balloon telescope, Astrophys. J. 898 (2020).

[24] R. Benhabib, R. Iwens, R. Jackson, Stability of distributed control for large flexible structures using positivity concepts, in: Guidance and Control Conference, AIAA, Boulder, CO, 1979.
[25] R.J. Benhabib, R.P. Iwens, R.L. Jackson, Stability of large space structure control systems using positivity concepts, J. Guid. Control 4 (1981) 487-494.

[26] Gawronski, Advanced Structural Dynamics and Active Control of Structures, Springer Science \& Business Media, 2004.

[27] J. Montel, F. Mirc, E. Pérot, I. Zenone, J.-M. Nicot, N. Bray, A. Gomes, J. Evrard, P. Tapie, P. Vola, B. Milliard, R. Grange, D. Schiminovich, Design and improvements of the Attitude Control System of the FIREBall balloon experiment, Proc. SPIE 9905 (2016), 99053I.

[28] J.P. Treilhou, J. Coutelier, J.J. Thocaven, C. Jacquey, Payload motions detected by balloon-borne fluxgate-type magnetometers, Adv. Space Res. 26 (2000) 1423-1426.

[29] P. Alexander, A. De La Torre, Uncertainties in the measurement of the atmospheric velocity due to balloon-gondola pendulum-like motions, Adv. Space Res. 47 (2011) 736-739.

[30] A. Preumont, Vibration Control of Active Structures, vol. 246, Springer International Publishing, 2018. 